\title{
Modeling and analysis of urban rail plug door system based on Petri net and SDG diagram
}

\author{
Wei $\mathrm{PENG}^{1,}{ }^{*}$, Erlin $\mathrm{LIU}^{1}$, Lihan $\mathrm{CHEN}^{1}$, and Dong $\mathrm{ZHAI}^{2}$ \\ ${ }^{1}$ School of Mechanical and Electrical Engineering, Lanzhou Jiaotong University, China \\ ${ }^{2}$ School of Automation and Electrical Engineering, Lanzhou Jiaotong University, China
}

\begin{abstract}
The on-the-road fault diagnosis of the urban rail train passenger compartment door is a weak field in the world research. At present, most of the fault diagnosis and monitoring models for door systems are based on the analysis of historical data. Under the background of continuous development and innovation of railroad crossing equipment, it is urgent to study the model of door system suitable for online monitoring and fault diagnosis. The modeling method combining SDG(signed directed graph) diagram and Petri net is adopted. The Petri net with improved conditional fuzzy time constraint is the first layer, and the SDG diagram is the second layer. Through the dynamic simulation and concurrent processing capability of Petri net, the dynamic process simulation of the system is carried out. At the same time, the SDG map and the Petri net are connected by means of standard tables; The SDG diagram is used to construct a hazard identification and fault mining for the causal relationship between related variables in a certain state of the library. Aiming at the urban rail passenger room plug door system, the model is established and the online safety monitoring hidden danger mining process of the model method in the urban rail plug door is analyzed.
\end{abstract}

\section{Introduction}

With the modernization of the city, urban rail transit plays an increasingly important role in the way the citizens travel. In addition to ensuring driving safety, the city-door guest room sliding door is also an important entrance for passengers. The distance between the urban rail operation site is short, and the working frequency of the door is high, which causes the damage and aging of the door components to cause frequent train door accidents during operation [1]. With the increasing speed of urban rail trains, it also brings great challenges to the monitoring and overhaul of the door. Therefore, the research on fault diagnosis and hidden danger mining of the door system is of great significance [2].

The on-the-road fault diagnosis of the door of the urban rail train passenger compartment is a weak field of research in the world ${ }^{[1]}$. At present, most researches on fault diagnosis methods for door systems are based on the analysis of historical data. Beijing Jiaotong University [3-4] used the door maintenance data as the support of the fault model, and used Petri net and its extension method to study the door fault diagnosis. In the logic

*Corresponding author: penw7763@foxmail.com 
relationship between the fault state and the determination of the weight, the randomness and uncertainty of the artificial experience are added to the model, which is separated from the simulated system and affects the accuracy of the model. Although the above related research has achieved very good results, but the real-time status information of the door system operation is not well utilized, there is still a problem of lack of real-time and effectiveness.

At present, there are not many methods to study the Hazard and Operability Analysis (Hazard and Operability Analysis) risk and operability analysis model of the dynamic discrete system. One is that Purdue University combined with the SDG (Signed Directed Graph) $[5,6]$. The method is used in industrial process control diagnostic systems; the other is a qualitative model established by the STARS software package development team of V. Bartolozzi et al [7], but this method is too complicated and requires a large model library. Wu Chongguang et al [8], who is mainly from Beijing University of Chemical Technology, deeply combined SDG and Petri net technology into HAZOP analysis of chemical systems, and achieved significant results. Based on the two-layer model of Purdue University's SDG and Petri nets, this paper uses the time-sharing Petri net and improves the conditional fuzzy time-constrained Petri net for the complex dynamic discrete system of the door system, and the door opening control system for the urban rail passenger compartment. The modeling analysis is carried out to demonstrate the effectiveness of the method in HAZOP analysis of the urban rail train sliding door control system.

\section{Petri net model}

\subsection{Time constraint}

References[9] put forward extended time-interval temporal logic, combined time constraints into Petri nets, and proposed several Petri models of temporal relations, which lay the time-line foundation for Petri nets linear reasoning. Reasoning based on time line can often find a better solution from another dimension [9]. Therefore, time factor is added to the fault hidden danger analysis model to provide a reference for the system risk analysis. In this paper, we set a constrained time slice $\pi(\mathrm{k})=\left[\mathrm{k}^{<}, \mathrm{k}^{-}, \mathrm{k}^{+}, \mathrm{k}^{>}\right]$(where $\mathrm{k}^{<} \leq \mathrm{k}^{-} \leq \mathrm{k}^{+}$ $\leq \mathrm{k}^{>}, \mathrm{k}$ is a real number, and $\mathrm{k}$ is determined by practical application.)

\subsection{Conditional fuzzy time}

Conditional fuzzy time Petri nets (Conditional Fuzzy Timing Petri Nets, CFTPN). In the fuzzy time Petri net workflow model, time constraints are introduced by assigning a constrained time slice $\pi(k)=\left[\mathrm{k}^{<}, \mathrm{k}^{-}, \mathrm{k}^{+}, \mathrm{k}^{>}\right]$. As shown in Figure 1 , we call $\left[\mathrm{k}^{-}, \mathrm{k}^{+}\right]$a "normal interval". If the transition occurs between $\left[t+\mathrm{k}^{-}, t+\mathrm{k}^{+}\right]$moments, the system is considered to be in normal operation. [ $\left.t+\mathrm{k}^{<}, t+\mathrm{k}^{-}\right]$and $\left[t+\mathrm{k}^{+}, t+\mathrm{k}^{>}\right]$are called "early warning zones". If the transition occurs between[ $\left.t+\mathrm{k}^{<}, t+\mathrm{k}^{-}\right]$and $\left[t+\mathrm{k}^{+}, t+\mathrm{k}^{>}\right]$, the transition can still be completed and the state transition can be completed, but the system will judge the current sub-healthy working state. [ $\left.-\infty, t+\mathrm{k}^{<}\right]$and $\left[t+\mathrm{k}^{>},+\infty\right]$ are called "fault zones". If the transition is in $\left[-\infty, t+\mathrm{k}^{<}\right]$and $\left[t+\mathrm{k}^{>},+\infty\right]$, the system is in fault state, that is, the transition can not end normally. Conditional fuzzy constraint time slice is shown in figure1 below: 


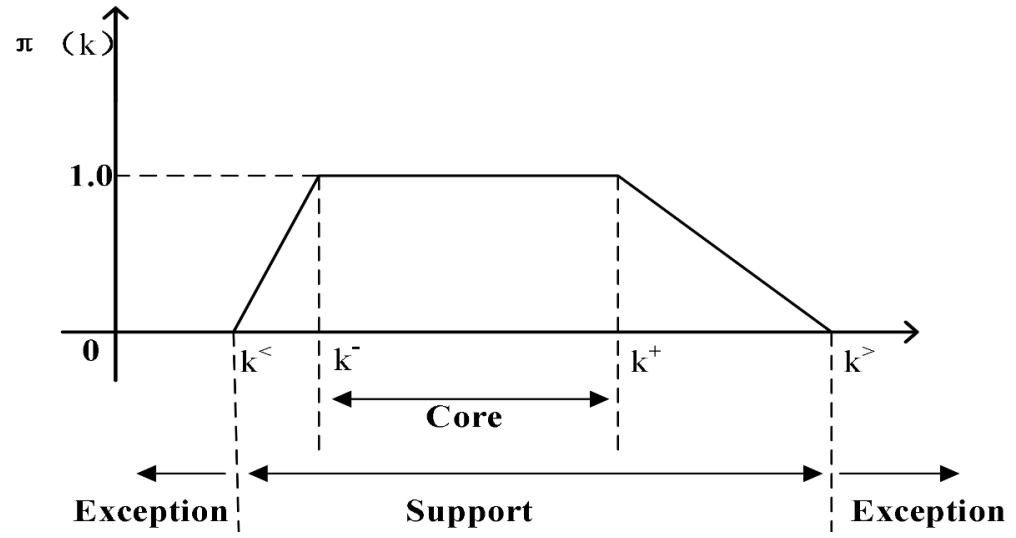

Fig. 1. Petri net constraint time slice trapezoid diagram.

\subsection{Timed Petri nets}

The definition of an improved conditional Fuzzy time Petri net (Conditional Fuzzy Timing Petri Nets, CFTPN) is a seven-tuple $\Sigma_{\mathrm{CFT}}=\left(\mathrm{S}, \mathrm{T} ; \mathrm{F}, \mathrm{I}, \pi, \mathrm{M}_{0}\right.$, Timed). Of which:

1) $\mathrm{S}$ is an extended repository, which represents the actual state of the system. Each repository contains Token and Token contains state information for fault diagnosis.

2) $\mathrm{T}$ is a transition, $\mathrm{F}$ is a directed arc between the repository of timed Petri nets and the transition, $\mathrm{I}$ is a suppression arc, and $\pi(\mathrm{k})$ is a conditional fuzzy constraint time slice to judge the time constraints of the repository to the transition.

3) $\mathrm{M}_{0}$ is the initial state identifier of the library $\mathrm{S}$ in $\Sigma_{\text {CFT. }}$ Timed $=\left(\mathrm{d}_{1}, \mathrm{~d}_{2}, \mathrm{~d}_{i}, \ldots, \mathrm{d}_{n}\right) \quad(\mathrm{n}>0)$ is a set of delay time parameters associated with the transition node or the repository node (That is, the time spent on the corresponding working steps of the system).

4) The delay time parameter is added to the library. For the corresponding initial marker $\mathrm{M}_{0}$, the initial vector is in formula (1):

$$
x_{i}\left(\tau_{0}\right)= \begin{cases}\tau_{0}, & p_{i}\left(\tau_{0}\right)=1 \\ \propto, & p_{i}\left(\tau_{0}\right)=0\end{cases}
$$

The delay vector Timed contains the delay time $\mathrm{d}_{i}$ of each repository. In the enabled vector, $\gamma(\tau)=\gamma_{0}(\tau), \gamma_{1}(\tau), \ldots, \gamma_{m}(\tau), \gamma_{j}(\tau)$,formula (2) indicates the earliest time that transition $\mathrm{T}_{j}$ can trigger:

$$
\gamma_{i}(\tau)=\max _{p_{i}}\left\{d_{i}+x_{i}\left(\tau_{k}\right)\right\}
$$

That is, when Token arrives at the repository $\mathrm{S}_{i}$, he should stay at least $\mathrm{d}_{i}$ time delay parameters to enable him to trigger the transition.

5)sequential workflow structure and parallel workflow structure in Time-constrained Petri Netse, as shown in figure 2 and figure 3.

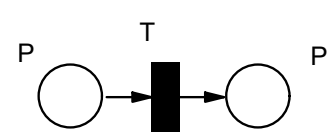

Fig. 2. sequential time constrained Petri nets.

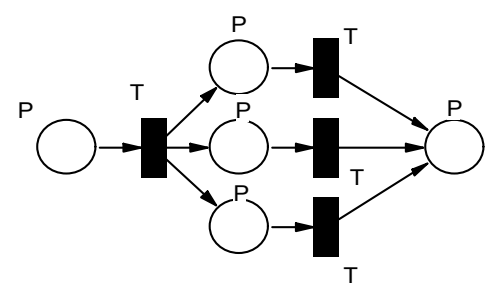

Fig. 3. parallel time constrained Petri nets. 


\subsection{Petri model of plug door}

The door system diagram is shown in figure 4 below. Taking the system state relation of the door opening process as an example, the door opening process can be divided into three major parts: the electrical control part, the EDCU control part and the mechanical action part. The main task of the electrical control part is to complete the train status identification to provide the starting power supply, and send out the opening signal and the driving motor signal from the console. The ECDU part is responsible for signal processing and control of the door movement process. The mechanical action layer is the mechanical action to complete the door opening. The whole process of opening the door requires the interaction of the three major parts, to achieve the train from parking to opening the entire action process.

The process simulation of the door system is carried out by analyzing the relationship between the hardware components of the door and the state of the system. The transfer of the state of each library is for a process with time recording. If the model monitors the time constraint of the relevant task process into the "warning zone" or "fault zone", the computer program corresponding to the analysis model will automatically enter the SDG diagnostic model from the real-time monitoring model of the Petri net. The information in the standard table corresponding to the neighboring library is compared to perform hidden danger mining and positioning.

For the time-constrained Petri net model of the door opening process of the door system, as shown in figure 5, the circle represents the library, and each library represents the current state of the door system related equipment or system. The library contains Token, and the value of Token will be updated in time with the working status of the system. The meaning of Token in the Petri net includes state information and control information, such as: the time when the system equipment completes the current task, the door signal is turned on, motor speed, temperature, etc. The box represents the transition and is used to indicate the transition of the state of the control system and the flow of information resources. The arc with the arrow indicates the flow relationship between the library and the transition, and the arc with the circle at the end indicates the conditional suppression relationship. The time constraint exception table corresponding to figure 5 is as shown in table1, and an example of the condition time of the door opening core process is listed.

The time from unlocking to normal operation is $3-5 \mathrm{~s}$. Take 5 seconds door opening time as an example. The time unit is ms. The typical time in the table is only for reference. The actual value can be obtained by the experiment of the specific door special PC software. The door controller will automatically record the corresponding time of each output in operation. 


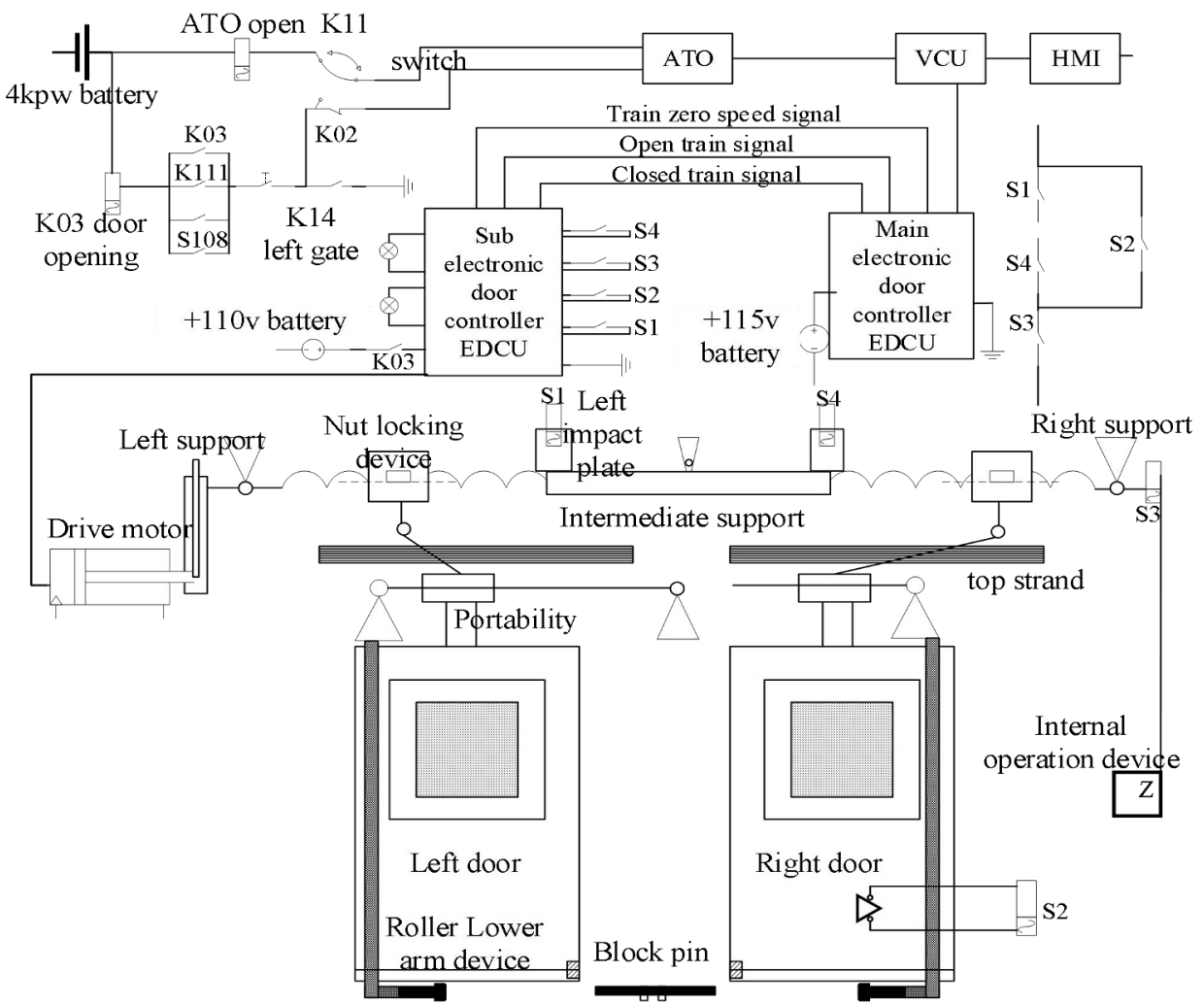

Fig. 4. structural diagram of door system.

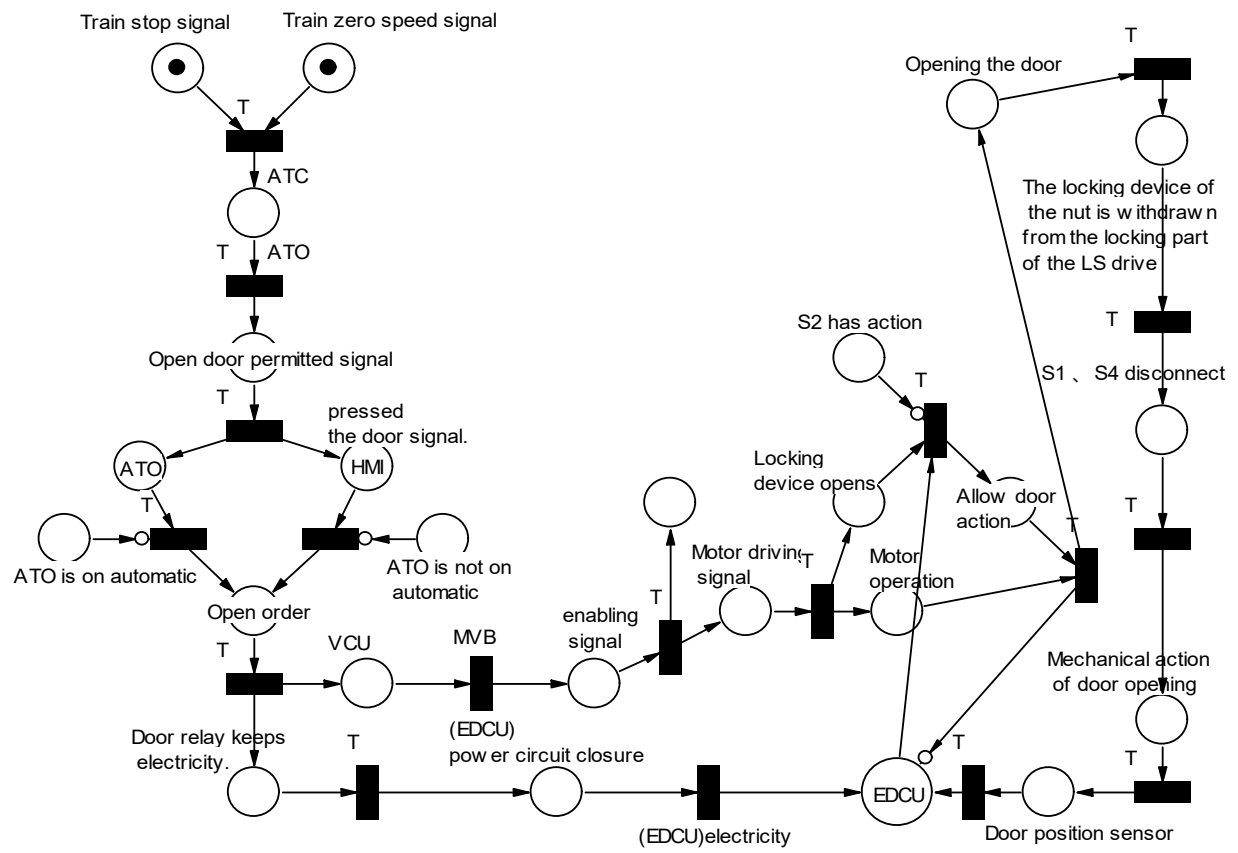

Fig. 5. Timed Petri net model for door opening process. 
Table 1. Petri network node conditional constraint time exception table for the core part of door opening.

\begin{tabular}{|l|c|c|c|c|c|}
\hline \multicolumn{1}{|c|}{ System state } & Number & $\begin{array}{c}\text { Normal } \\
\text { working } \\
\text { hour (ms) } \\
{\left[t+\mathrm{k}^{-}, t+\mathrm{k}^{+}\right]}\end{array}$ & $\begin{array}{c}\text { Early warning } \\
\text { zone time } \\
(\mathrm{ms}) \\
{\left[t+\mathrm{k}^{<}, t+\mathrm{k}^{-}\right] \text {] }} \\
{\left[t+\mathrm{k}^{+}, t+\mathrm{k}^{>}\right]}\end{array}$ & $\begin{array}{c}\text { Fault zone time } \\
(\mathrm{ms}) \\
{\left[-\infty, t+\mathrm{k}^{<}\right] \text {or }} \\
{\left[t+\mathrm{k}^{>},+\infty\right]}\end{array}$ & $\begin{array}{c}\text { Normal } \\
\text { delay time }\end{array}$ \\
\hline $\begin{array}{l}\text { Order in driver's } \\
\text { cab to open door }\end{array}$ & $\mathrm{A}$ & $0-500$ & $\begin{array}{c}0-100,500- \\
600\end{array}$ & $\mathrm{t}<0, \mathrm{t}>500$ & 500 \\
\hline Keep the relay alive & $\mathrm{B}$ & $500-1000$ & $\begin{array}{c}500-600,900- \\
1000\end{array}$ & $\mathrm{t}<500, \mathrm{t}>500$ & 500 \\
\hline $\begin{array}{l}\text { EDCU motor } \\
\text { driving signal }\end{array}$ & $\mathrm{C}$ & $1000-1500$ & $\begin{array}{c}1000-1100, \\
1400-1500\end{array}$ & $\mathrm{t}<1000, \mathrm{t}>1500$ & 500 \\
\hline $\begin{array}{l}\text { EDU power circuit } \\
\text { closure }\end{array}$ & $\mathrm{D}$ & $1500-2000$ & $\begin{array}{c}1500-1600, \\
2900-2000\end{array}$ & $\mathrm{t}<1500, \mathrm{t}>2000$ & 500 \\
\hline Signal lamp & $\mathrm{E}$ & $2100-2300$ & $\begin{array}{c}2100-2200, \\
2200-2300\end{array}$ & $\mathrm{t}<2100, \mathrm{t}>2300$ & 200 \\
\hline $\begin{array}{l}\text { The locking device } \\
\text { opens }\end{array}$ & $\mathrm{F}$ & $2300-2500$ & $\begin{array}{c}2300-2400, \\
2400-2500\end{array}$ & $\mathrm{t}<2300, \mathrm{t}>2500$ & 200 \\
\hline $\begin{array}{l}\text { Lock device exit } \\
\text { LS lock section }\end{array}$ & $\mathrm{G}$ & $2500-2800$ & $\begin{array}{c}2500-2600, \\
2700-2800\end{array}$ & $\mathrm{t}<2500, \mathrm{t}>2800$ & 300 \\
\hline S S S4break & $\mathrm{H}$ & $2800-3000$ & $\begin{array}{c}2800-2900, \\
2900-3500\end{array}$ & $\mathrm{t}<2800, \mathrm{t}>3500$ & 200 \\
\hline $\begin{array}{l}\text { Open } \\
\text { mechanical action }\end{array}$ & $\mathrm{I}$ & $3000-4900$ & $\begin{array}{c}2500-3000, \\
3000-3500\end{array}$ & $\mathrm{t}<2500, \mathrm{t}>3500$ & 1900 \\
\hline $\begin{array}{l}\text { Door open position } \\
\text { sensor trigger }\end{array}$ & $\mathrm{J}$ & $4900-5000$ & $\begin{array}{c}4500-4600, \\
4900-5000\end{array}$ & $\mathrm{t}<4500, \mathrm{t}>5000$ & 100 \\
\hline
\end{tabular}

\section{The Petri net and SDG two-layer model connection}

The connection mode between the Petri net and the SDG two-layer model is in the form of a standard table. The standard table corresponds to the system command status or device information of the Token installation in each library, and the corresponding system information will have corresponding standard values. Each electrical quantity or information transfer library corresponds to a standard table. The deviation caused by the operation or fault will be reflected to the equipment status and command information; when the time deviation of the transition in the first model of the Petri net exceeds the deviation of the reasonable area, the automatic calculation of the SDG layer is completed by comparing the error standard table of the relevant variables. Reasoning and analysis of adverse consequences and early fault risk mining reasoning.

\section{Failure analysis}

\subsection{Establishment of SDG Model of EDCU}

In the SDG model, a circle is often used to represent the node, which represents a certain control quantity in practice. The control amount exceeds the upper limit, the lower limit and the normal state are represented by $(+,-, 0)$ respectively. Where the line represents the relationship between the control and other related variables. This paper takes the entering SDG model analysis as an example when the bound time slice of EDCU in the timeconstrained Petri net model is abnormal. The SDG exception analysis model for EDCU is shown in figure 6. 


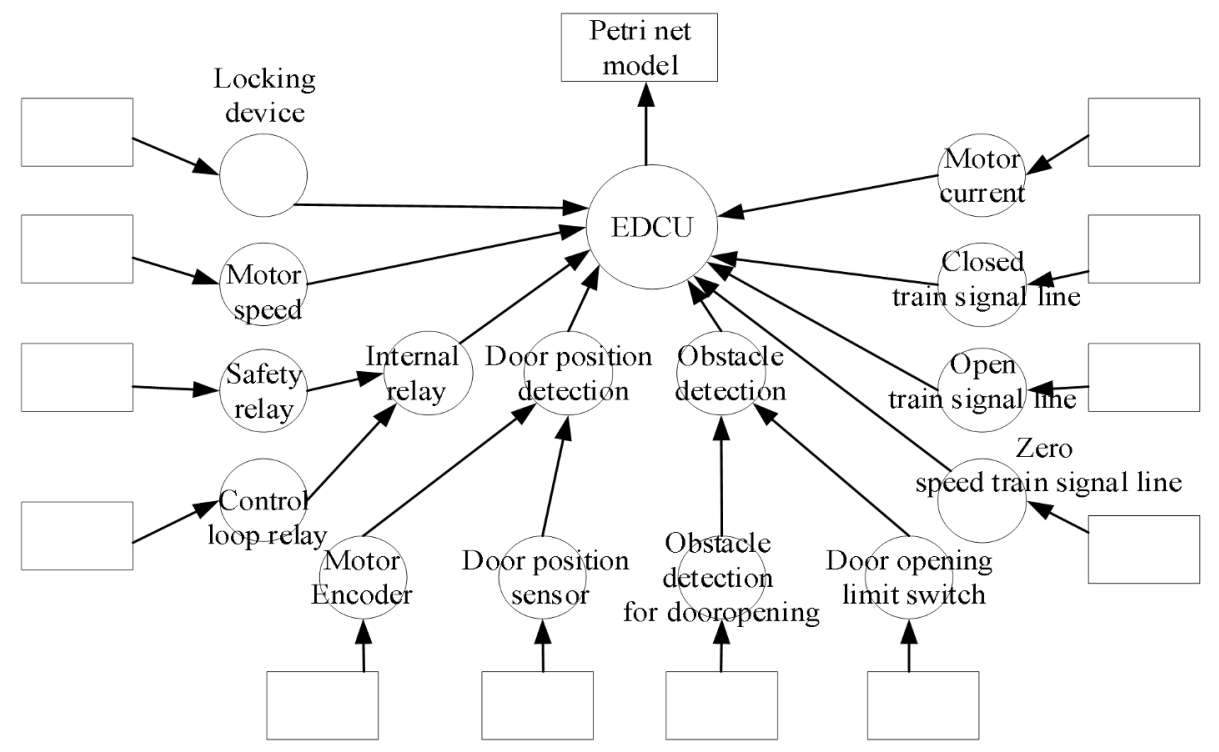

Fig. 6. SDG model diagram of EDCU.

As shown in Figure 6, if there is an abnormal alarm in the EDCU, there are two types of situations. On the one hand, there is a hidden fault inside the EDCU itself, and on the other hand, the node connected to the EDCU device is abnormal.

1) If only the EDCU device time constraint exception, EDCU internal self-query.

2) If the time constraint transition of EDCU equipment associated with node also has an abnormal alarm, the fault correlation variables of SDG model can be correlated together to carry out a more comprehensive hidden trouble mining. The abnormal position can be found from the subdivision parameter error, and the hidden danger location and adverse consequence analysis can be carried out accordingly. This hidden trouble location method, which does not rely on mathematical statistics and fault tree analysis, is based on the influence relationship between the related variables while the system is running, and has a simpler query path, which makes the query of abnormal parts more real-time and efficient. Table 2 is a standard table of deviation analysis related to EDCU in the library. It lists the system information standard tables corresponding to the equipment in the event of EDCU anomalies.

Table 2. Standard tables for EDCU related deviation analysis in the library.

\begin{tabular}{|l|l|l|}
\hline \multicolumn{1}{|c|}{ name } & Value statement (Unspecified is Boolean) & standard value \\
\hline Safety relay & 1-Get electricity;0-Loss of electricity & 1.0 \\
\hline Control circuit relay & 1-Get electricity;0-Loss of electricity & 1.0 \\
\hline Motor encoder & 1- regular work ;0-unusual & 1.0 \\
\hline Left door position & Floating point type,unit mm & 0 -n \\
\hline Right door position & Floating point type,unit mm & 0 -n \\
\hline Motor speed & Integer,unit r/min & 0 -n \\
\hline $\begin{array}{l}\text { Motor working current nominal } \\
\text { value }\end{array}$ & Floating point type,unit mA & $\mathrm{A}_{1} \leq \mathrm{x} \leq \mathrm{A}_{2}$ \\
\hline Door barrier pressure detection & Floating point type,unit N & $70 \leq \mathrm{F} \leq 150$ \\
\hline Door limit switch & $\begin{array}{l}\text { 1-Open the door in place;0-Close the door } \\
\text { in place }\end{array}$ & 1.0 \\
\hline Zero speed line & 1-Zero speed;0-Speed other & 1.0 \\
\hline
\end{tabular}




\begin{tabular}{|l|l|l|}
\hline Open train line & 1-Open the door to allow;0-No opening & 1.0 \\
\hline Closed train line & $\begin{array}{l}\text { 1-Close the door to allow;0-No door } \\
\text { closing }\end{array}$ & 1.0 \\
\hline Locking device & $\begin{array}{l}\text { 1-Unlock anomaly }(\mathrm{T} \leq(3-0.5) \mathrm{s}) \\
0 \text {-Unlock success }((3-0.5) \mathrm{s} \leq \mathrm{T} \leq(3+0.5) \mathrm{s}) ; \\
-1-\text {-Unlock anomaly }((3+0.5) \mathrm{s} \leq \mathrm{T})\end{array}$ \\
\hline
\end{tabular}

Note: $A_{1}$ and $A_{2}$ are the standard value of a factory's comprehensive test when it comes out of the factory.Normal mechanical resistance of door $\leq 70 \mathrm{~N}$, Maximum anti-extrusion pressure $\leq 150 \mathrm{~N}$.

\subsection{Analysis of hidden trouble}

Next, the abnormal situation of the door controller node is taken as an example for analysis. When the model Petri net enters the SDG layer, the token information in the library will be compared with the standard values in Table2. If the anomaly is found, the hidden danger will be dug and the adverse consequences will be analyzed:

Monitoring gets the following information: $\mathrm{B}=[500 \mathrm{~ms}, 1100 \mathrm{~ms}], \mathrm{C}=[1000 \mathrm{~ms}$, $2000 \mathrm{~ms}], \mathrm{D}=2100 \mathrm{~ms}, \mathrm{E}=[2300 \mathrm{~ms}, 2500 \mathrm{~ms}], \mathrm{F}=[2500 \mathrm{~ms}, 2700 \mathrm{~ms}], \mathrm{G}=[2700 \mathrm{~ms}$, $3200 \mathrm{~ms}], \mathrm{H}=[3200 \mathrm{~ms}, 3400 \mathrm{~ms}], \mathrm{I}=4000 \mathrm{~ms}$

Petri net layer judges that the above information is abnormal, and the corresponding codes in table 1 indicate the time or duration interval that first captures the corresponding state of the system. No action of the locking device F was observed. It is assumed that the door should start at $3000 \mathrm{~ms}$ under normal working conditions, but there is no mechanical action of the door, and the motor will automatically stop the rotation. Find out the running equipment that may break down.

Analysis of diagnostic results:

(1)From the above information, it can be concluded that the node with the first time exception is $\mathrm{B}$, and the subsequent nodes also have the time correlation delay, which does not exclude the possibility of failure in the following steps.

(2)According to the definition 4 in $\Sigma$ CFT $=$ (S,T;F,I, $\pi, M 0$,Timed):Add a delay parameter condition to the library to calculate whether the delay time used for each step is longer than the time taken in Table 1 to work properly: $\mathrm{B}=[500 \mathrm{~ms}, 1100 \mathrm{~ms}]=600, \mathrm{C}=$ $[1000 \mathrm{~ms}, 2000 \mathrm{~ms}]=1000, D=2100 \mathrm{~ms}, \quad E=[2300 \mathrm{~ms}, 2500 \mathrm{~ms}]=200, \quad F=[2500 \mathrm{~ms}$, $2700 \mathrm{~ms}]=200, \mathrm{G}=[2700 \mathrm{~ms}, 3000 \mathrm{~ms}]=500, \mathrm{H}=[3200 \mathrm{~ms}, 3400 \mathrm{~ms}]=200, \mathrm{I}=4000 \mathrm{~ms}$

By comparing with the normal working time of Table 1, it can be concluded that the time spent by nodes B and $\mathrm{G}$ is slightly exceeded, and the $\mathrm{C}$ and I nodes seriously exceed the normal working time. The $\mathrm{C}$ node is the motor drive signal from the door controller EDCU. If $\mathrm{C}$ is abnormal, the subsequent I corresponds to the door opening mechanical action, which is delayed or stopped. It can be initially determined that the fault is located in the door controller EDCU.

After judging and screening out the abnormal nodes from the Petri net layer, the system model is transferred into the SDG diagram model analysis. According to figure 6, the SDG model of EDCU controller was constructed, and relevant control variables affecting the normal output of motor drive signals of EDCU node $\mathrm{C}$ were found, namely, G lock device signal and obstacle pressure detection signal.These two control variables may lead to control delay or do not emit motor drive signal. At this point, more specific abnormal parameters and locations can be found by comparing the state information of token in the library with the nominal values in the standard table of deviation analysis of table 2 . Table 3 and table 4 below show all hidden dangers of lock device and obstacle pressure detection in the fault analysis and analysis of adverse consequences: 
Table 3. Analysis of abnormal hidden danger in door barrier pressure detection.

\begin{tabular}{|c|l|}
\hline \multirow{4}{*}{ Adverse consequences: } & $\begin{array}{l}\text { Open door pressure detection exceeds the upper limit of } \\
\text { normal pressure range, } \\
\text { Cause door to be unable to open } \\
\text { EDU sends obstacle alarm. }\end{array}$ \\
\hline \multirow{4}{*}{$\begin{array}{c}\text { The hidden trouble which leads to } \\
\text { the abnormal pressure of obstacle } \\
\text { is: }\end{array}$} & Component deformation stagnation \\
\cline { 2 - 2 } & Insufficient lubrication: Long guide column,feed screw nut \\
\cline { 2 - 2 } & Arm screw and guide rail interference \\
\cline { 2 - 2 } & Lower guideway deformation \\
\hline & Upper guideway misalignment \\
\hline & Nut accessory failure \\
\hline
\end{tabular}

Table 4. Analysis of abnormal hidden danger of locking device.

\begin{tabular}{|c|l|}
\hline Adverse consequences: & $\begin{array}{l}\text { An abnormal unlock device will cause the door to } \\
\text { fail to unlock in 3s, Door alarm }\end{array}$ \\
\hline \multirow{3}{*}{$\begin{array}{c}\text { The hidden trouble that leads to the } \\
\text { abnormal locking device is: }\end{array}$} & EDCU internal module failure \\
\cline { 2 - 2 } & Wire rope loosening \\
\cline { 2 - 2 } & Fork interference \\
\cline { 2 - 2 } & Unlocked locator fracture \\
\hline
\end{tabular}

Therefore, the diagnosis and analysis results will be recommended to overhaul the door obstacle detection device and locking device. In summary, the adverse consequences and hidden trouble location of abnormal occurrence of related nodes are listed in table 3 and 4 above, which provides great convenience for the monitoring and maintenance of the door system.

\section{Conclusion}

In the maintenance of the door system, the actual situation urgently needs the online realtime fault hidden danger monitoring means, targeted maintenance and overhaul of the subhealth door with abnormal conditions. Turning planned repairs into state repairs is a hot trend in current door fault diagnosis. The analysis model of this paper can effectively distinguish the working state of the door, and can quickly find all relevant abnormal parts, which provides a real-time and effective analysis model for the development of online early fault detection system. What needs to be done in the follow-up work is to combine the analysis model with the computer program and transplant it to the MCU controller for testing, to achieve a very efficient online fault detection system.

\section{References}

1. Liu Guangwu, Cai Changjun, Chen Gang, Yang Lingzhi and Xing Zongyi. (2015). Reliability analysis of plug door system based on fault tree and fuzzy inference petri net. Mechanical Manufacturing and Automation (3), 146-149.

2. Shen Bibo, Qi Wei, Ye Yangdong and Jia Limin. (2016). A tc-cpn-based method for diagnosing the fault of urban railroad car door faults. Journal of the China Railway Society, 38(4), 51-58.

3. Liu Jing. (2014). Application of improved fuzzy Petri nets in the fault diagnosis of the door system of urban rail train passenger compartment. (Doctoral dissertation, Beijing Jiaotong University). 
4. Qin Yong, Jia Limin, Fu Yunyu, Su Wei, Gong Ling and Ye Yangdong et al. (2015). Method for locating hidden danger of train door system based on probabilistic petri net. CN105046061A.

5. Maurya, M. R., Rengaswamy, R. and Venkatasubramanian, V. (2004). Application of signed digraphs-based analysis for fault diagnosis of chemical process flowsheets. Engineering Applications of Artificial Intelligence, 17(5), 501-518.

6. Maurya, M. R., Rengaswamy, R.and Venkatasubramanian, V. (2006). A signed directed graph-based systematic framework for steady-state malfunction diagnosis

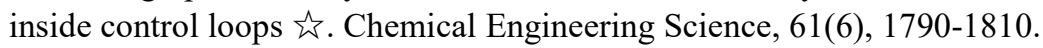

7. Bartolozzi, V., Castiglione, L., Picciotto, A. and Galluzzo, M. (2000). Qualitative models of equipment units and their use in automatic hazop analysis. Reliability Engineering \& System Safety, 70(1), 49- 57.

8. Wang Wei, Zhang Baker and Wu Chongguang. (2007). An improved batch process hazop analysis model. Computer Simulation, 24(2), 57-61.

9. Shen Bibo. (2015). Research on CPS fault diagnosis method based on time-constrained self-controlled Petri net. (Doctoral dissertation, Zhengzhou University). 